\title{
Diez años de estudio del comportamiento higrotérmico de la cubierta del Museo del Prado
}

\section{Ten years study of the hygrothermal behaviour of the Prado museum's roof}

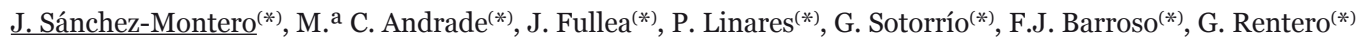

\section{RESUMEN}

Debido a unas filtraciones detectadas al terminar la renovación de las cubiertas del Museo del Prado se decidió instalar sensores en sus diversas capas, cuyo diseño no es tradicional. Después de más de diez años de registro, los sensores de agua líquida muestran la acumulación de humedad en la capa de impermeabilización líquida. Sin embargo, no se ha detectado la presencia de agua líquida en las capas inferiores. Los sensores de humedad relativa muestran la presencia de condensaciones locales. Los sensores de temperatura muestran la capacidad aislante de las diferentes capas de la cubierta. Y los sensores de deformación muestran un incremento de deformación continuo con el tiempo, posiblemente debido a la fluencia del conjunto. Como conclusión del estudio, se puede afirmar que el comportamiento de la cubierta ha sido correcto ya que no se ha detectado la aparición de agua líquida en las capas internas de la cubierta.

Palabras clave: Sensores; monitorización; cubiertas; Museo del Prado.

\section{ABSTRACT}

In the current paper we present the results of the monitoring of the hygrothermal behaviour during the last ten years of the El Prado Museum roof. Sensors were installed in the south roof of the museum after it underwent mayor repair following a leak over a very important exhibition hall (the one holding Velazquez's Las Meninas painting). The liquid water sensors reveal that water is retained at the waterproofing layer. These sensors do not show water presence in lower layers of the roof. The humidity sensors show condensation when the ambient temperature drops. The temperature sensors show the insulating capacity of the different layers of the roof, which effectively attenuate the outside temperature changes. The strain sensors placed on the liquid waterproofing layer show a constant increase of strain with time. In summary, the repaired roof can be considered to perform as intended and no more leaks have been detected.

Keywords: Sensor; monitoring; roof; Prado Museum.

(*) Instituto de Ciencias de la Construcción Eduardo Torroja (IETcc-CSIC). Madrid (España). Persona de contacto/Corresponding author: javier.sanchez@csic.es (J. Sánchez-Montero)

Cómo citar este artículo/Citation: Sánchez-Montero, J., Andrade, M. ${ }^{a}$ C., Fullea, J., Linares, P., Sotorrío, G., Barroso, F. J., Rentero, G. (2014). Diez años de estudio del comportamiento higrotérmico de la cubierta del Museo del Prado. Informes de la Construcción, 66(535): e027, doi: http://dx.doi.org/10.3989/ic.12.121.

Licencia/License: Salvo indicación contraria, todos los contenidos de la edición electrónica de Informes de la Construcción se distribuyen bajo una licencia de uso y distribución Creative Commons Reconocimiento no Comercial 3.o. España (cc-by-nc). 


\section{INTRODUCCIÓN}

El edificio que actualmente alberga el Museo Nacional del Prado fue diseñado por el arquitecto Juan de Villanueva en 1785 por encargo de Carlos III y en un principio estaba destinado a acoger la Academia de Ciencias Naturales. Después de diversas vicisitudes, Fernando VII destinó el edificio a pinacoteca. El Museo Nacional del Prado abrió por primera vez sus puertas al público en noviembre de 1819.

En el edificio se han realizado diversas reformas a lo largo de su historia (1). La última gran intervención y que afectó a las cubiertas es de los años 1995 y 1997 en los que se sustituyeron la mayoría de las mismas con los objetivos de proporcionar unas cubiertas funcionalmente adecuadas y de homogeneizar su aspecto con una protección única a base de planchas de plomo. A raíz de estas intervenciones y con motivo de la gotera aparecida en la sala que entonces albergaba el cuadro de Las Meninas de Velázquez, en el año 2001 el Instituto de Ciencias de la Construcción Eduardo Torroja (IETcc-CSIC) fue requerido por el entonces Ministerio de Educación y Cultura para estudiar la estanqueidad de la solución constructiva adoptada (2).

La solución actual es una cubierta plana invertida compuesta por capas de plomo, capa de impermeabilizante de aplicación líquida (SIL), caucho y mortero (Figura 1). La pendiente de la cubierta es del $1,5 \%$.

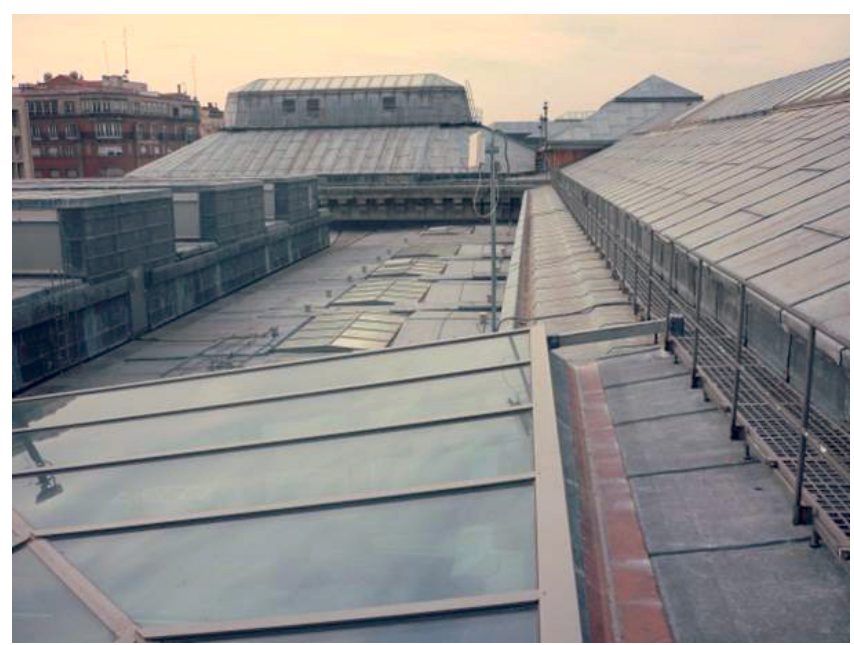

Figura 1. Vista de la actual cubierta del Museo Nacional de El Prado.

Existe una amplia bibliografía sobre el control de ambiente interior en edificios históricos, sin embargo, es escasa la literatura sobre el control de aspectos relacionados con las cubiertas. Existe un grupo italiano que es muy activo en el estudio de edificios históricos y que emplea técnicas no destructivas, como la emisión acústica para para el control de reparaciones (3), la fisuración de la estructura (4) (5) o del daño (6) y las técnicas de monitorización en $3 \mathrm{D}$ junto con las simulaciones por elementos finitos (7). El IETcc realizó un estudio previo para determinar el tipo de sensores que, embebidos en las diferentes capas de la cubierta, permitieran suministrar una información permanente del comportamiento higrométrico de la misma. Los sensores debían ser diseñados para cumplir los siguientes requisitos: I) que pudieran ser embebidos en puntos estratégicos de la cubierta, II) que no produjeran alteraciones en la misma, y III) que se asegurara su estabilidad, es decir, que fueran durables (8) y no se deterioraran al estar embebidos en materiales de construcción (9) (10).

En el presente trabajo se muestran los resultados de los últimos diez años de la monitorización de una parte representativa de la cubierta sur del Museo del Prado. Los resultados obtenidos ponen de manifiesto la utilidad de ciertos tipos de sensores que, embebidos en la cubierta, permiten por una parte confirmar si la solución constructiva adoptada es correcta y, por otra, detectar un posible fallo bien sea de diseño, de construcción o por deterioro de los materiales empleados.

La metodología desarrollada puede ser de gran interés en aquellas cubiertas de edificios singulares, como el aquí descrito, donde se requiera conocer anticipadamente un posible fallo de estanquidad, con el fin de tomar las medidas oportunas que impidan un riesgo no deseado por la gravedad de los daños que se pueden producir.

\section{METODOLOGÍA EXPERIMENTAL.}

\subsection{Sensores}

Los sensores empleados en las cubiertas son de cuatro tipos: Sensores de temperatura (tipo A), Sensores de agua líquida (tipo B), Sensores de humedad relativa (tipo C) y Galgas extensométricas (tipo D).

Los sensores de temperatura (Sensor tipo A) son sensores del tipo resistivo. Su exactitud es de $\pm 1{ }^{\circ} \mathrm{C}$ y su temperatura de funcionamiento de $-50 / 500{ }^{\circ} \mathrm{C}$. En algunos puntos se ha instalado un sensor que además de medir la temperatura en todos los rangos también mide la humedad relativa.

Los sensores de agua líquida (sensor tipo B) permiten detectar la presencia de agua con una simple medida de potencial. La respuesta del sensor a la presencia de agua es relativamente rápida. El potencial llega a valores entorno a los $850 \mathrm{mV}$ en aproximadamente uno o dos minutos después de humedecer el sensor.

Los sensores de humedad relativa (sensor tipo C) son del tipo capacitivo y se han utilizado dos modelos. El primero de ellos mide humedad relativa en un intervalo de $\mathrm{o} / 100 \%$ no condensante, su precisión es del $5 \%$ (al $100 \%$ HR) y su temperatura de operación es de $-20 / 80^{\circ} \mathrm{C}$. Dispone, en el mismo encapsulado, de un sensor de temperatura que, con una precisión de $\pm 0,75{ }^{\circ} \mathrm{C}$, facilita la compensación de la medida en todo el margen de temperatura. El otro sensor de humedad relativa que ha sido utilizado posee un intervalo de medida de $0 / 100 \%$ no condensante, una exactitud del $2 \%$ y la temperatura de funcionamiento es $\mathrm{de}-4 \mathrm{O} / 85^{\circ} \mathrm{C}$.

La deformación se registra mediante la colocación de galgas extensométricas (sensor tipo D). Estas galgas, que son adecuadas para materiales poliméricos, tienen una longitud de $5 \mathrm{~mm}$, una resistencia de $120 \Omega$ y un factor de galga de 2,09, siendo su sensibilidad transversal de un $0,1 \%$ y la compensación de temperatura para $5 \times 10^{-6} /{ }^{\circ} \mathrm{C}$.

Las medidas se llevan a cabo mediante una unidad de adquisición y conmutación de datos, Data Logger. Este dispositivo dispone de una memoria interna para almacenamiento de los datos, lo cual hace posible su funcionamiento de forma 
independiente sin necesidad de estar permanentemente conectado a un ordenador. Además la configuración de la toma de datos se mantiene incluso si ocurren cortes de electricidad. Los datos almacenados se descargan en un ordenador portátil mediante el empleo de un programa que también permite la configuración de los 60 canales de medida del Data Logger. El error del Data Logger, que incluye la medición, la conmutación y la conversión del transductor, es inferior al $0,1 \%$ en las medidas de voltaje, temperatura y resistencia.

\subsection{Cubierta}

Para una mejor comprensión de la colocación y comportamiento de los sensores, a continuación se describe brevemente la composición por capas de la cubierta. En la Figura 2 se muestra la distribución de las capas que conforman la cubierta plana.
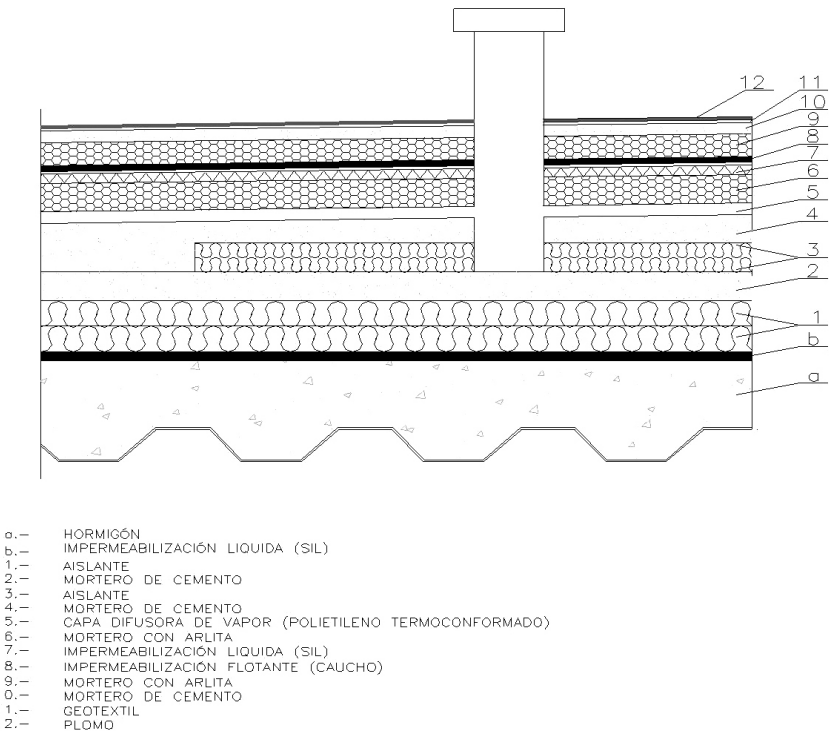

Figura 2. Sección correspondiente a la cubierta plana.

La cubierta plana se encuentra dividida en paños (véase Figura 3) en cuyo centro se sitúan lucernarios de cristal para iluminar las salas situadas bajo estas cubiertas. El paño de seguimiento se encuentra en la cubierta sur donde se distinguen dos zonas de medida dentro del mismo paño (véase Figura 4).

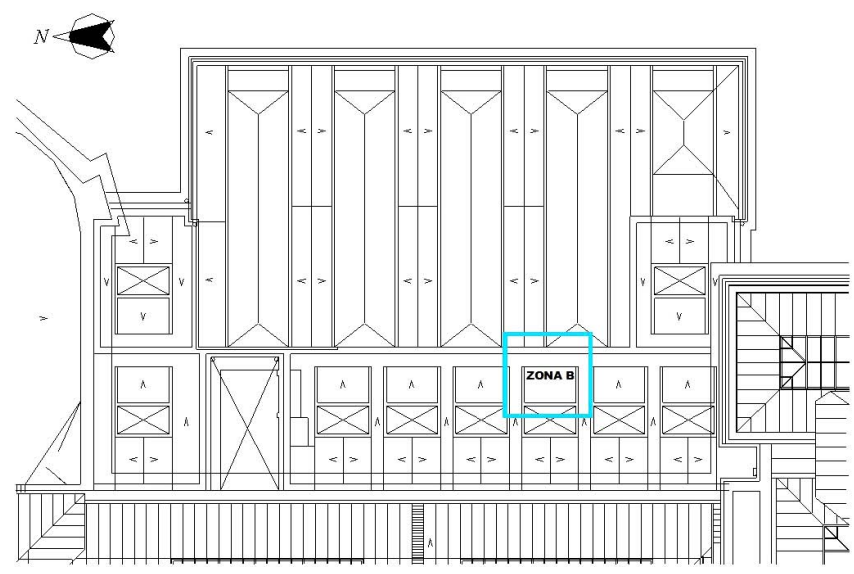

Figura 3. Localización del paño de seguimiento en la cubierta sur.

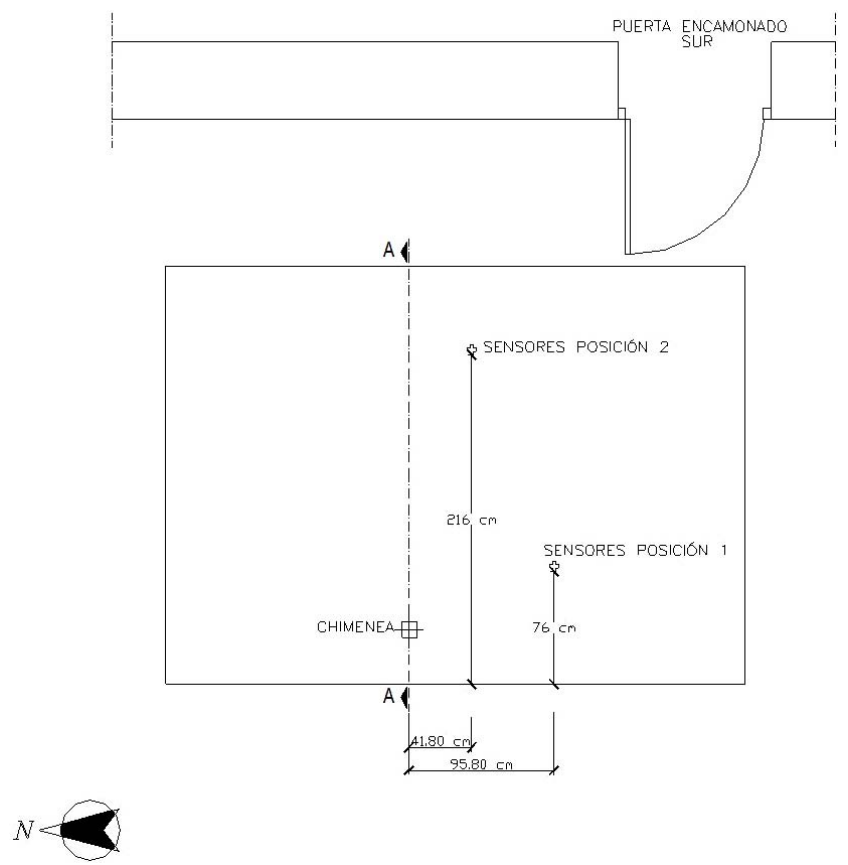

Figura 4. Posiciones de los sensores dentro del paño.

\subsection{DISTRIBUCIÓN DE LOS SENSORES EN LA CUBIERTA}

La colocación de los sensores se llevó a cabo durante la realización de las obras de reforma de la cubierta. Los sensores de agua líquida y temperatura se colocaron entre cada una de las capas de la cubierta y en dos posiciones distintas, una cercana a la chimenea y otra más alejada. Las galgas extensométricas se situaron sobre la capa de SIL con el fin de monitorizar la continuidad de la misma. Los sensores de humedad relativa se situaron en diversas posiciones en las capas de impermeabilización y debajo de la capa superficial de plomo. En la Tabla 1 se muestran todos los sensores instalados en la cubierta. La letra se corresponde con el tipo de sensor, el primer número indica una de las dos posiciones posibles según la Figura 4, y

Tabla 1. Tipos de sensores instalados.

\begin{tabular}{|c|l|c|l|}
\hline SENSOR & TIPO SENSOR & SENSOR & \multicolumn{1}{|c|}{ TIPO SENSOR } \\
\hline A01 & Temperatura & B26 & Agua líquida \\
\hline Bo1 & Agua líquida & A17 & Temperatura \\
\hline A12 & Temperatura & B17 & Agua líquida \\
\hline B12 & Agua líquida & C17 & Humedad relativa \\
\hline A22 & Temperatura & D17 & Galga extensométrica \\
\hline B22 & Agua líquida & A27 & Temperatura \\
\hline A13 & Temperatura & B27 & Agua líquida \\
\hline B13 & Agua líquida & D27 & Galga extensométrica \\
\hline A14 & Temperatura & A18 & Temperatura \\
\hline B14 & Agua líquida & B18 & Agua líquida \\
\hline A24 & Temperatura & C18 & Humedad relativa \\
\hline B24 & Agua líquida & A28 & Temperatura \\
\hline A15 & Temperatura & B28 & Agua líquida \\
\hline B15 & Agua líquida & C28 & Humedad relativa \\
\hline A25 & Temperatura & A19 & Temperatura \\
\hline B25 & Agua líquida & B19 & Agua líquida \\
\hline A16 & Temperatura & C19 & Humedad relativa \\
\hline B16 & Agua líquida & A29 & Temperatura \\
\hline C16 & Humedad relativa & B29 & Agua líquida \\
\hline A26 & Temperatura & C29 & Humedad relativa \\
\hline & & & \\
\hline
\end{tabular}


el segundo número indica la capa de la cubierta sobre la que se encuentra. En la Figura 5 se muestra la disposición de los sensores que se encuentran más próximos a la chimenea.

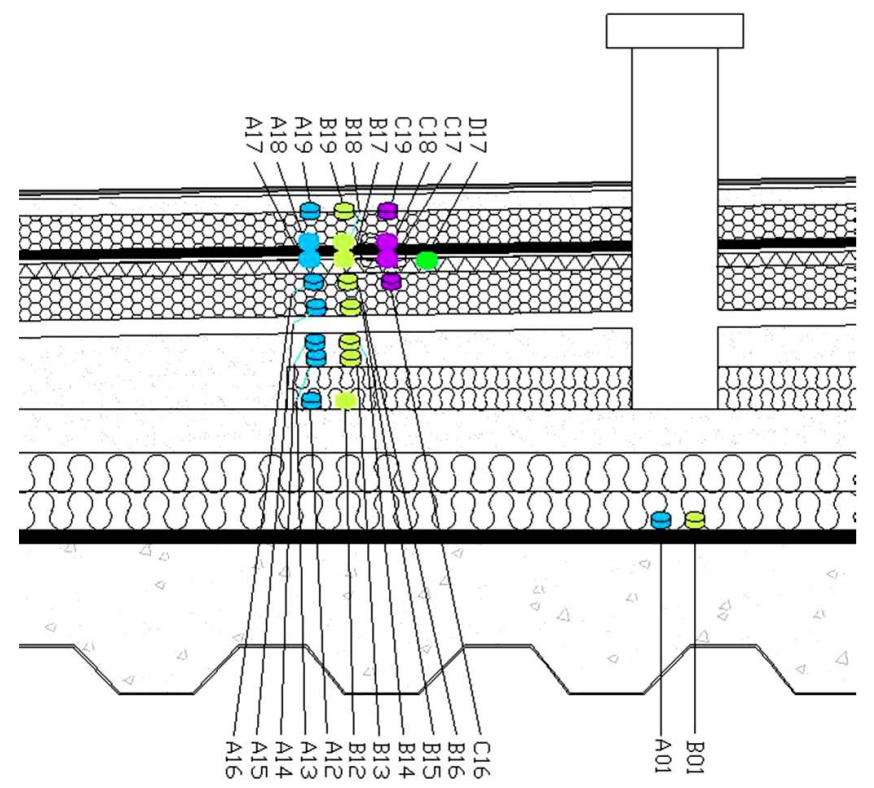

Figura 5. Disposición de los sensores más cercanos a la chimenea, posición 1.

\section{RESULTADOS Y DISCUSIÓN}

En la Figura 6 se muestra el registro completo de la medida de temperatura para uno de los dos sensores situados debajo de la capa de plomo. En la gráfica se puede observar los ciclos anuales de temperatura y las variaciones diarias. En esta posición es en la que se alcanzan las temperaturas más extremas, superándose $\operatorname{los} 50^{\circ} \mathrm{C}$ en verano y llegando a valores bajo $\mathrm{O}^{\circ} \mathrm{C}$ en invierno.

A19

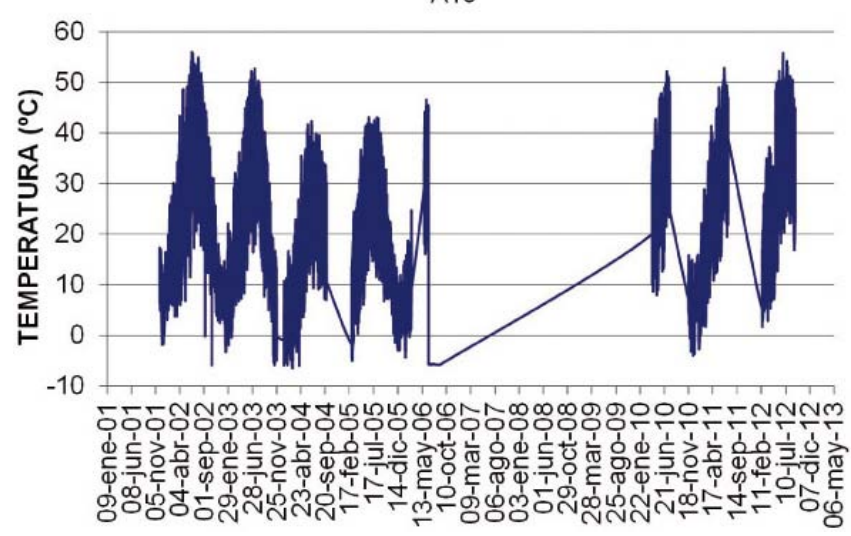

Figura 6. Sensor de temperatura A19, situado en la posición 1 y sobre la capa 9.

En la Figura 7 se muestra la variación de la temperatura en dos sensores situados a distintas alturas. El sensor que se encuentra más cerca de la superficie de la cubierta (A15) muestra grandes oscilaciones diarias, mientras que el sensor más interno que se ha instalado (A01) se mantiene prácticamente constante.

Si se tienen en cuenta los sensores instalados en todas las capas de la cubierta es posible observar la atenuación de la va-

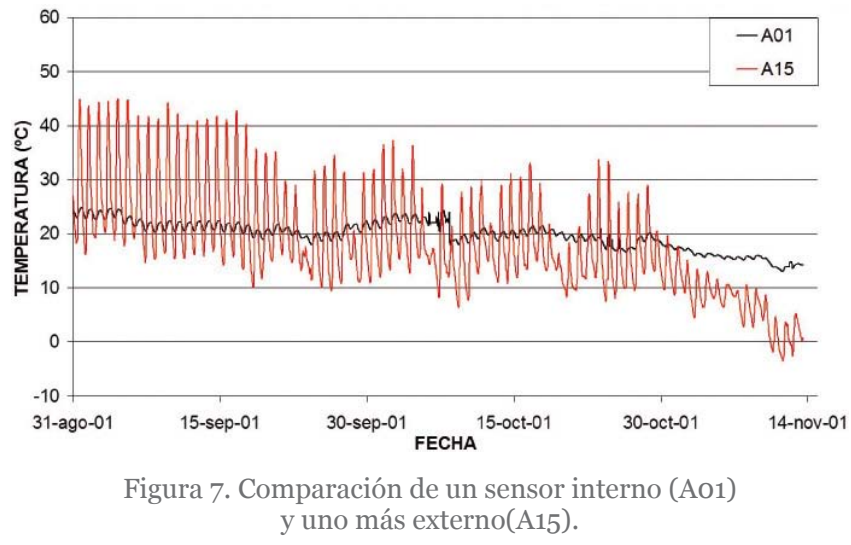

riabilidad diaria de la temperatura con la profundidad (véase Figura 8). Los sensores más externos sufren una gran variación de temperatura influida por la temperatura ambiente y la insolación, mientras que a medida que aumenta la profundidad las variaciones de temperatura se hacen más suaves. Estos parámetros son un indicador de la buena ejecución de la obra y el aislamiento logrado.
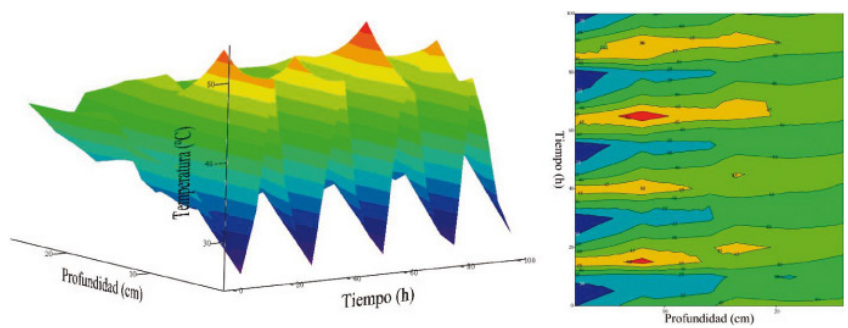

Figura 8. Gradiente de temperaturas en la cubierta y su variación diaria.

Los sensores de humedad relativa utilizados presentan el principal problema de que si se saturan luego su recuperación es muy lenta al producirse condensaciones sobre el sensor, por ello es necesario interpretar los datos teniendo en cuenta que una vez alcanzadas elevadas humedades la histéresis de los sensores es muy grande. Según Andrade y otros (11), la humedad relativa en el interior de un hormigón se mantiene en valores superiores al 60\%, por lo que es posible que aparezcan condensaciones en ciertas posiciones, como por ejemplo entre la capa de impermeabilización flotante y el mortero aligerado con arlita. En la Figura 9 se muestran los valores de humedad relativa frente a la temperatura. La tendencia general es que la humedad relativa disminuye al aumentar la temperatura

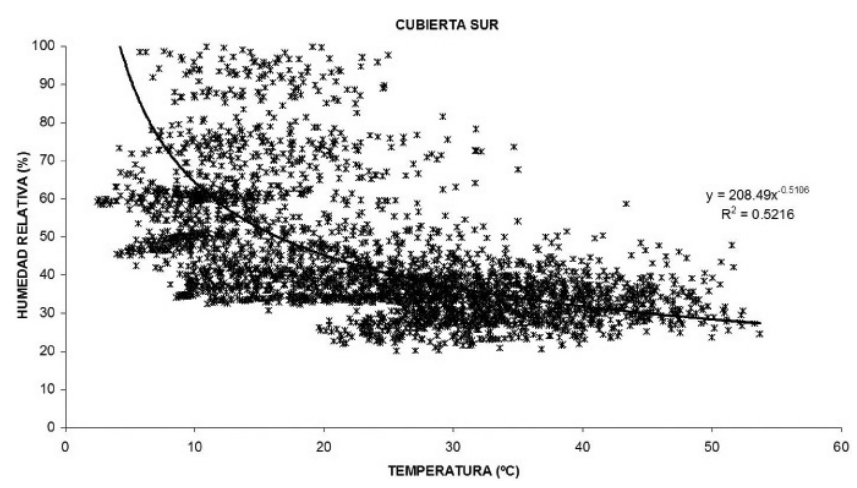

Figura 9. Sensores de humedad relativa. 
y, además, este comportamiento no se ve influenciado ni por las épocas de sequía ni tampoco por las lluvias, pero hay mucha dispersión de resultados posiblemente debido a los ciclos de histéresis antes mencionados de los sensores.

Las galgas extensométricas monitorizan el comportamiento de la capa de SIL para comprobar que no se produce la rotura de la misma, rotura que conllevaría la pérdida de estanqueidad de la cubierta. Las deformaciones medidas han ido aumentando a lo largo del tiempo (tal vez por fluencia del sistema completo) y parecen mantenerse estables durante el último periodo de tiempo (Figura 10). En el registro de las galgas existe una discontinuidad que se puede atribuir a una sobretensión en la alimentación de las mismas. El valor absoluto es de unas $200 \mu \varepsilon$, o lo que es lo mismo, una deformación de 0,02\%. Además de la tendencia general, existe una variación dimensional de la capa de impermeabilización generada por los cambios de temperatura diaria (Figura 11).

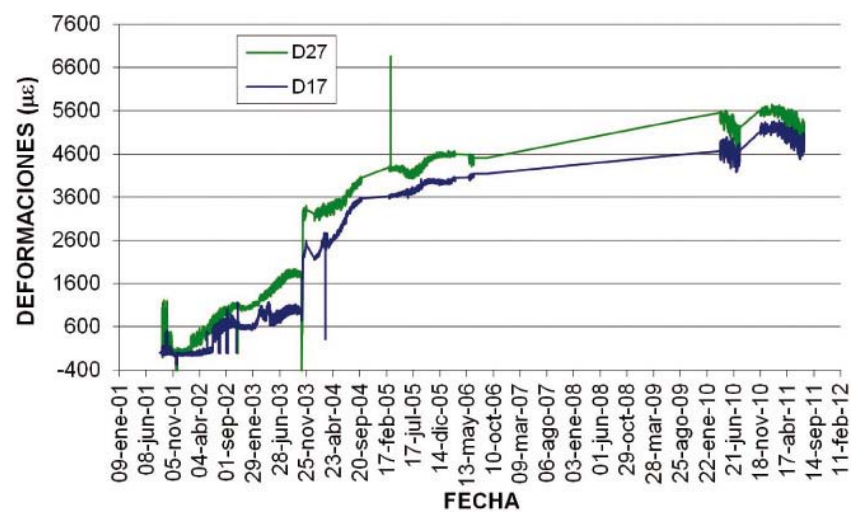

Figura 10. Galgas extensométricas instaladas en la impermeabilización líquida.

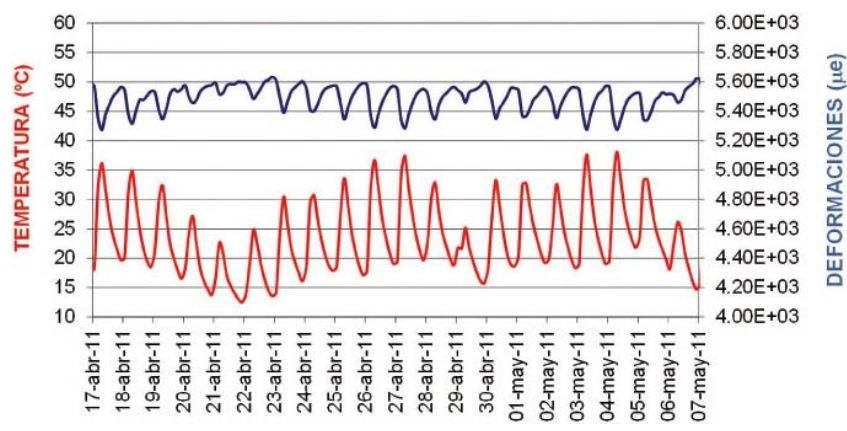

Figura 11. Efecto de la variación de temperatura (sensor A27) sobre la deformación de la capa de SIL (sensor D27).

Los sensores de agua líquida se han instalado en todas las capas de la cubierta durante la construcción de la misma. Estos sensores han sido probados en el laboratorio y están en fase de ser patentados. En la Figura 12 se muestra el registro de uno de los sensores de agua líquida durante su periodo de instalación (sensor B17). Este sensor permaneció a la intemperie sobre la impermeabilización líquida hasta la colocación de la impermeabilización flotante. Se puede observar cómo se activa en presencia del agua de lluvia, ya que su exposición coincidió con la aparición de precipitaciones, hasta el momento en el que se instaló la impermeabilización flotante. A partir de este momento deja de estar expuesto a la lluvia y el valor de potencial vuelve a valores próximos a cero.

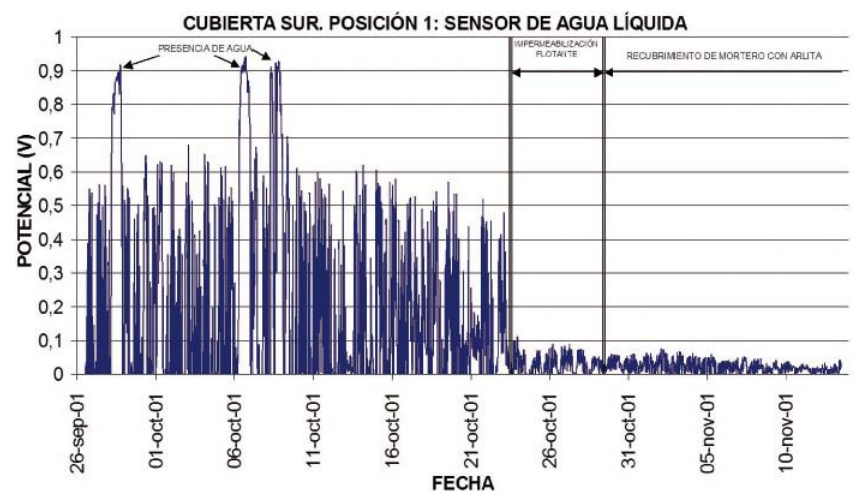

Figura 12. Registro del Sensor de agua líquida B17.

En la Figura 13 se muestran los valores medios registrados en los diferentes sensores. Todos los sensores se encuentran en valores de potencial inferiores al rango de detección de agua líquida, o,8 - o,6 mV, es decir, no detectan la presencia de agua líquida. Los diferentes rangos de valores entre los sensores se deben a la posición en la que se encuentran. Los sensores que se encuentran en contacto con el mortero tienen un potencial mayor que el resto de los sensores debido a la alta humedad relativa del mortero, que puede llegar a producir condensaciones en algunas localizaciones (11).

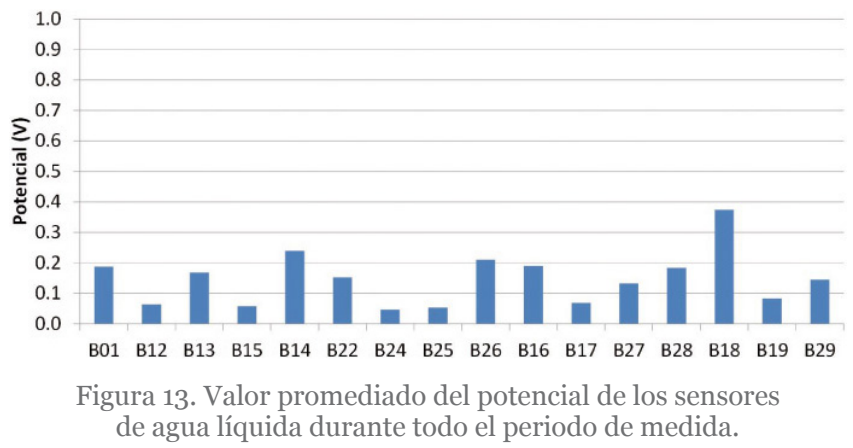

\section{CONCLUSIONES}

La conclusión general a la fecha actual es que el funcionamiento de la cubierta es correcto, ya que los sensores de agua líquida muestran que no se ha producido aporte de humedad desde el exterior a las capas interiores de la cubierta, concretamente, a las que se encuentran debajo de la impermeabilización líquida.

Durante los más de diez años de registro se ha analizado la evolución de todos los sensores que se encuentran en las diferentes capas de la cubierta. Durante este periodo se ha puesto de manifiesto que algunos sensores han fallado, aunque muy pocos, en especial no han funcionado los de humedad relativa.

\section{AGRADECIMIENTOS}

Los autores desean mostrar su agradecimiento a la Gerencia de Infraestructuras y Equipamientos del Ministerio de Cultura y al Museo Nacional del Prado. 


\section{REFERENCIAS}

(1) de Luxán, M. (1989). Restauración de la iluminación natural. Informes de la Construcción, 40(399): 5.

(2) Sanchez, J., Andrade, C., Fullea J. (2010). Hydrothermal monitoring using embedded sensors of the actual roof system of the Prado Museum. Construction and Building Materials, 24(12): 2579-2589, doi: http://dx.doi.org/10.1016/j. conbuildmat.2010.05.018.

(3) Anzani, A., Binda, L., Carpinteri, A., Lacidogna, G., Manuello, A. (2008). Evaluation of the repair on multiple leaf stone masonry by acoustic emission. Materials and Structures, 41(6): 1169-1189, doi: http://dx.doi.org/10.1617/s11527-0079316-z.

(4) Carpinteri, A., Invernizzi, S., Lacidogna, G. (2009). Historical brick-masonry subjected to double flat-jack test: Acoustic emissions and scale effects on cracking density. Construction and Building Materials, 23(8): 2813-2820, doi: http:// dx.doi.org/10.1016/j.conbuildmat.2009.03.003.

(5) Carpinteri, A., Invernizzi, S., Lacidogna, G. (2007). Structural assessment of a 17th-century masonry vault with acoustic emissions and numerical techniques. International Journal of Architectural Heritage 1(2): 214-226, doi: http://dx.doi. org/10.1080/15583050701287649.

(6) Carpinteri, A., Lacidogna, G. (2007). Damage evaluation of three masonry towers by acoustic emission. Engineering Structures, 29(7): 1569-1579, doi: http://dx.doi.org/10.1016/j.engstruct.2006.08.008.

(7) Carpinteri, A., Lacidogna, G., Invernizzi, S., Manuello, A., Binda, L. (2009). Stability of the vertical bearing structures of the Syracuse Cathedral: experimental and numerical evaluation. Materials and Structures, 42(7): 877-888, doi: http:// dx.doi.org/10.1617/s11527-008-9429-z.

(8) Andrade, C., Sanchez, J., Fullea, J., Rebolledo, N., Tavares, F. (2012). On-site corrosion rate measurements: 3D simulation and representative values. Materials and Corrosion, 63(12): 1154-1164.

(9) Martínez, I., Andrade, C. (2009). Examples of reinforcement corrosion monitoring by embedded sensors in concrete structures. Cement and Concrete Composites, 31(8): 545-554, doi: http://dx.doi.org/10.1016/j.cemconcomp.2009.05.007.

(10) Feliu, S., Gonzalez, J. A., Andrade, C. (1996). Techniques to Assess the Corrosion Activity of Steel Reinforced Concrete Structures. American Society for Testing and Materials Special Technical Publication. pp. 107-118, doi: http://dx.doi. org/10.1520/STP16970S.

(11) Andrade, C., Sarria, J., Alonso, C. (1999). Relative humidity in the interior of concrete exposed to natural and artificial weathering. Cement and Concrete Research, 29(8): 1249-1259, doi: http://dx.doi.org/10.1016/Sooo8-8846(99)o0123-4. 